\title{
Based on 2D Barcode Technology to Design of Wolfberry Products Traceability System
}

\author{
Wang Chen, Li Jianbei, Zhang Xuejian, Wen Shuping, Li Shucheng \\ Agricultural Science and Technology Information Research Institute, Ningxia Academy of Agriculture and Forestry Sciences, Yinchuan, \\ Ningxia, China
}

Email address:

elanor2001@126.com (Wang Chen)

To cite this article:

Wang Chen, Li Jianbei, Zhang Xuejian, Wen Shuping, Li Shucheng. Based on 2D Barcode Technology to Design of Wolfberry Products Traceability System. Internet of Things and Cloud Computing. Vol. 4, No. 3, 2016, pp. 19-22. doi: 10.11648/j.iotcc.20160403.11

Received: April 10, 2016; Accepted: June 1, 2016; Published: June 2, 2016

\begin{abstract}
The main purpose of this paper is to study the application of 2D barcode in the design of the Chinese wolfberry product traceability system. Through the design of Chinese wolfberry product traceability system, the application of 2D barcode technology, the application of 2D barcode to identify agricultural products, so as to improve the traceability of Chinese wolfberry products. The results confirmed that the application of $2 \mathrm{D}$ barcode technology to design the product traceability system, can improve the traceability of Chinese wolfberry products, compared with the previous increase of $6 \%$, play a positive impact. Using 2D barcode technology to Ddetermine agricultural products, not only to enhance the design level of Chinese wolfberry product traceability system, but also effectively play the application value, improve the traceability of wolfberry products.
\end{abstract}

Keywords: 2D Barcode Technology, Product Traceability System, Chinese Wolfberry, System Design

\section{Introduction}

A 2D (two-dimensional) barcode is a graphical image that stores information both horizontally and vertically. As a result of that construction, 2D codes can store up to 7,089 characters, significantly greater storage than is possible with the 20-character capacity of a unidimensional barcode. 2D barcode is no stranger to the public. It is widely used in products or leaflets and personal business cards. 2D barcode. The products detail instructions page can be directly linked by scanning sweep code software. 2D barcodes are also known as quick response codes because they enable fast data access. 2D barcodes are often used in conjunction with smart phones. The user simply photographs a 2D barcode with the camera on a phone equipped with a barcode reader. The reader interprets the encoded URL, which directs the browser to the relevant information on a Web site. This capability has made 2D barcodes useful for mobile marketing. Some 2D barcode systems also deliver information in a message for users without Web access. The following article is about the application of 2D barcode in the design of the Chinese wolfberry product traceability design system.

\section{2D Barcode Technology}

\subsection{Technical Features}

2D barcode technology is one kind of two-dimensional bar code. It has lots of features such as: a large number of stored information, high confidentiality, wide coding, high reliability decoding, powerful correction, cheap cost and so on. The convenience of mobile data also leads into the innovational trend time from one dimensional bar code to QR bar code recently, so it's the great trend to use 2D barcode for anti-faking and anti- fleeing goods.

\subsection{Role of $2 D$ Barcode}

Meanwhile, 2D barcode also plays a role in traceability. If manufacturers will use 2D barcode, first they should re-code some products information to the background of 2D barcode, such as producing area, production batch, date and fundamental information of products, and the city where it will be sent. After recording, then scan the 2D barcode that has been done into the system with scan-gun. Such products can be shipped, ensure the products traceability. 


\section{Requirement Analysis of Designing the Chinese Wolfberry Products Traceability}

Based on the 2D barcode technology, during the design of the Chinese wolfberry product traceability system, the following items can be analyzed by it, the Chinese wolfberry markets functional system, knowing about the clients of using the Chinese wolfberry whose requirement on its system design, visiting the clients and experts of clients, linking the clients' practical operating environment, operating course and operating requirement, comparing the problems of clients hand in. In the system design, make sure that rural committees supervise the products and take measurement. The suppliers, materials, manufacturers, principals and products including the Chinese wolfberry from which it's forming to its flow direction can be controlled exactly by manufacturers. If there is something trouble in products quality during producing, it can quickly be located in every link of products, and controlled its flow direction. Distributors in Chinese wolfberry products management, the whole distribution process of the Chinese wolfberry products can be managed and controlled completely effectively and safely. Consumers on anti-faking of the Chinese wolfberry products can scan 2D barcode many times and connect with WEB platform back to the food traceability information after scanning. In the whole system design, also need to guarantee the scalability and expansibility of the Chinese wolfberry products traceability system, make them have considerable information processing capacity on communication, computer and network equipments, reserve a interface of software and hardware to maintain update and expand easily and to adjust to the requirement of future development.

\section{Design is Based on the 2D Barcode Technology of the Chinese Wolfberry Products Traceability System}

\subsection{The General Design of System}

In this system, first, 2D barcode technology application represents identification of every Chinese wolfberry product which is in the traceability. Then, stick the label of 2D barcode traceability on the finished products' package of enterprise, register products information of enterprises and checking data, re-code the products link during producing. Finally, the consumers can scan 2D barcode with mobile phone to check the result of products traceability, locate and get the symbolic imagine, the forming array of dark and light color modules identified as " 0 " and " 1 "; read format information, indentify version information, confirm symbolic version. According to the rule of module arrangement, read sign character and reply information of data and correct the wrong notes. The dealing with the 2D barcode should be packed in the $2 \mathrm{D}$ barcode decoder, there is a decoding way called String decode (2D barcode image), and the 2D barcode image is the photo to need identify, after then turn to character string. If using $2 \mathrm{D}$ barcode image, it passes on the Bit map object when creating the 2D barcode image object. There are three same size position direction patterns in $2 \mathrm{D}$ barcode. These three position direction patterns will be in the top left corner, top right corner and left bottom of 2D barcode note without turning. (Figure 1) the image figure which composed by three position direction patterns.

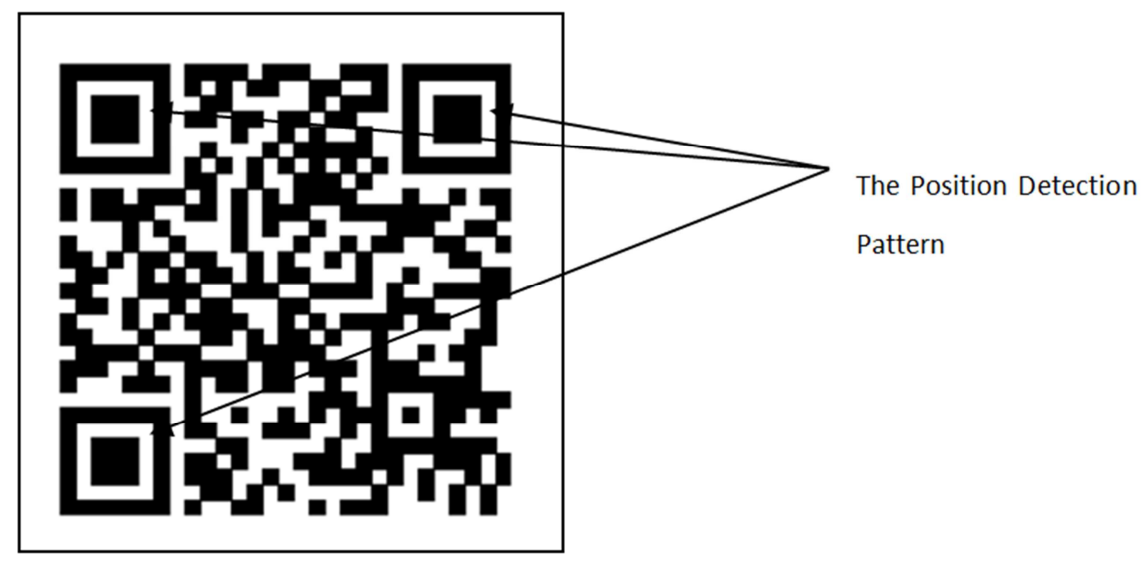

Figure 1. The Position Detection Pattern of 2D barcode.

To realize protection in the bottom, to become the identifying code for consumers to indentify, there is evidence to trace the real products and no evidence to trace the fake products. The consumers feel at ease when the every products of the Chinese wolfberry can be guaranteed their authenticity and reliability. If "the traceability system" can be a code of practice or can be set up the whole protection of the Chinese wolfberry industry base, which can prevent deteriorating the
Chinese wolfberry industry.

\subsection{System Functional Design}

As to the traceability system functional design part of the Chinese wolfberry products, there are three modules to design, rural committee, manufacturer and traceability module. As the following figure 2 : 


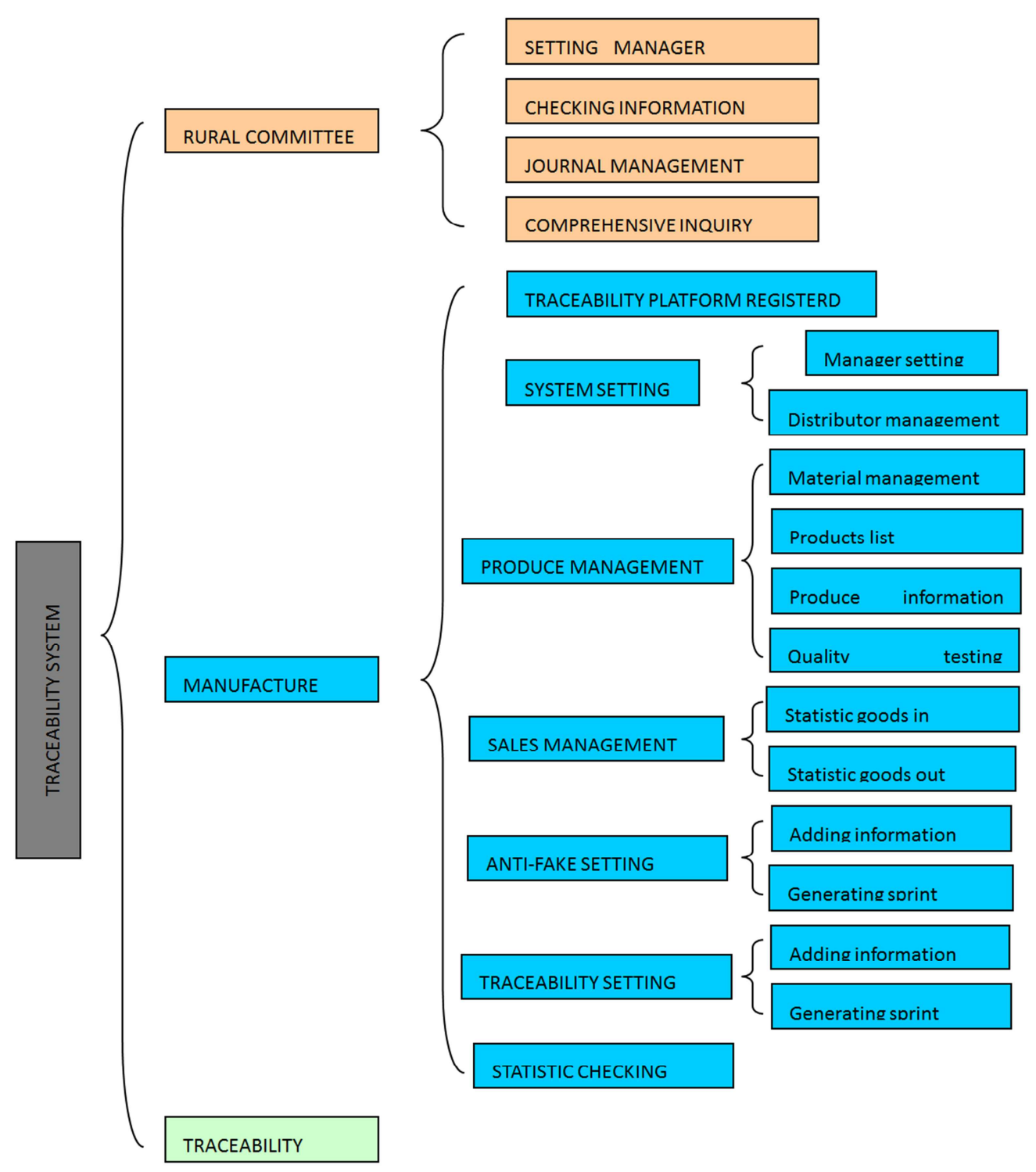

Figure 2. Structure of system function.

Rural committee: the role of system management and supervision, in charge of producing supervision of the food industry; there is function for setting the managers, checking information, looking through journal and comprehensive inquiry.

Manufacturer: the role of the food circulation data enter in the system, in charge of producing material into commodity sales; there is function for registered information, manufacturer system management, producing management, distributor management and comprehensive checking.

Traceability function: the manager of system platform, in charge of setting up system network (including WEB service, data acquisition equipment, consumer go online with mobile phone), make sure people should trace the Chinese wolfberry products through this system.

\subsection{Technical Building Designs}

Using B/S structure based on Net 2.0 platform, using Ajax,
Web service technology connecting with SQL Server 2005 database development. Data acquisition equipment is used Windows CE system. Database and WEB service can be hot standby, guarantee data safety. Network firewalls can prevent the data breaking by Hacker.

Meanwhile, there are some requirements in system interface design:

WEB platform client: use IE 6 browser, the number of connecting is more than 1000 , the time of response is less $100 \mathrm{~ms}$.

Data acquisition equipment: two-dimension code indentify module, wireless module, $2 \mathrm{G} / 3 \mathrm{G}$ online module, Windows $\mathrm{CE}$ operating system, dial online connecting time is less $30 \mathrm{~s}$, two-dimension code time of decoding is less $100 \mathrm{~ms}$, data cervices response time is less $3 \mathrm{~s}$, the time of standby is more than $72 \mathrm{~h}$, continues working time is more than $8 \mathrm{~h}$, working temperature is less 60 degree, working humidity is less than $80 \%$, meet the standard of waterproof and dustproof IP54, it's strong enough to protect from $1.8 \mathrm{~m}$ falling down. 
Print equipment: industrial grade two-dimension code print, continuously work for $24 \mathrm{~h}$ one more year, daily print quantity is more than 50000 pages, consumers can take photos online with smart mobile phone (the phone system supported: Windows Mobile, Symbian, Android, IOS).

\subsection{Realize the Traceability}

During the Chinese wolfberry products traceability system design, the 2D barcode will be turned black bar code with white background by taking gray histogram tool and using iteration method to choose properly value to deal with $2 \mathrm{D}$ barcode with binaryzation. Confirm 2D barcode position direction pattern to locate the bar code, after rotation to level to get the data of bar code, and then to grayscale code.

ToGrayScale:

\{

$$
\begin{aligned}
& \text { for (int } \mathrm{y}=0 ; \mathrm{y}<\text { image[0].Length; } \mathrm{y}++ \text { ) } \\
& \{ \\
& \text { for (int } \mathrm{x}=0 ; \mathrm{x}<\text { image.Length; } \mathrm{x}^{++} \text {) } \\
& \text { \{ } \\
& \text { int } \mathrm{r}=\text { image }[\mathrm{x}][\mathrm{y}]>>16 \& 0 \mathrm{xFF} \text {; } \\
& \text { int } \mathrm{g}=\text { image }[\mathrm{x}][\mathrm{y}]>>8 \text { \& } 0 \mathrm{xFF} \text {; } \\
& \text { int } \mathrm{b}=\text { image }[\mathrm{x}][\mathrm{y}] \& 0 \mathrm{xFF} \text {; } \\
& \text { int } \mathrm{m}=(\mathrm{r} * 30+\mathrm{g} * 59+\mathrm{b} * 11) / 100 \text {; } \\
& \text { image }[\mathrm{x}][\mathrm{y}]=\mathrm{m} \text {; } \\
& \text { \}\} }
\end{aligned}
$$

The consumers should scan 2D barcode, then they can read the information that manufacturers are ready to give the consumers, the information of the Chinese wolfberry products traceability can be checked.

\section{Efficiency Analysis of Using 2D Barcode Technology in the Chinese Wolfberry Product Traceability System}

During the Chinese wolfberry product traceability system design, using 2D barcode technology in design the Chinese wolfberry product traceability system, the traceability can be promoted. It can be promoted more $20 \%$ than before, it has the positive inference. It can make sure all serious information of the Chinese wolfberry product from getting its materials to producing and quarantine inspection to record into the database of $2 \mathrm{D}$ barcode traceability platform. In order to convenient consumers to know more about the information, to make manufacturers realize the whole-process monitor and check exactly, the code figure is printed and bounded to the system design. When using 2D barcode technology, a safe and convenient platform of two-dimension bar code traceability will be supplied between merchant and consumer. Merchant can manage well the product and check exactly and deal well with the food problem by using the 2D barcode traceability system, to reduce loss. The consumers can know more about the Chinese wolfberry products' information by installing equipment of mobile identification, scanning the products of
$2 \mathrm{D}$ barcode to play the positive application roles.

\section{Conclusion}

To sum up all above all, during the Chinese wolfberry product traceability system design, based on the 2D barcode technology, the 2D barcode traceability application can be realized. During the Chinese wolfberry products producing, to mark the agriculture products by using 2D barcode technology, not only can promote the level of traceability system design, but also can play the valuable role of application efficiently. It can also promote the traceability of the Chinese wolfberry products and exert a positive influence.

\section{Acknowledgements}

Fund project: "The science and technology innovation pilot fund project of Ningxia Academy of Agriculture and Forestry Sciences", the Chinese wolfberry quality security traceability coding system design and implement (project tasks number: NKYJ-14-15).

\section{References}

[1] Zhao Li, Xing Bin, Li Wenyong et al. Agricultural product quality and safety traceability system based on the recognition of mobile phone two-dimensional bar code [J]. Journal of agricultural machinery, 2012, 43 (7): 124-129.

[2] Chen Xi, Li Jianguo, Ma Jin equality. Information management of Ningxia wolfberry product traceability system based on QR code technology $[\mathrm{J}]$. Ningxia agriculture and forestry science and technology, 2012, 53 (12): 216-218.

[3] Tang Yanwei, Guo Zhonghua, Li Shuqing et al. Design and implementation of the traceability system for the quality and safety of Muslim beef and mutton based on QR code [J]. Jiangsu agricultural science, 2014, (10): 395-397.

[4] Lan longhui, Qiu rongzu etc.. Based on the 2D barcode, fruits and other agricultural products traceability system preliminary design. Logistics technology, 2014, (9): 417-419.

[5] Zhao Zhuo, Stewart he Zhiyong and other. QR code in the agricultural products supply chain traceability system research and application $[\mathrm{J}]$. Agricultural Library Information Journal, 2010, 22 (1): 33-36. (11): 5-8.

[6] Wen Bin, Liang Peng, Luo, etc. based on the QR 2D barcode and data aggregation agricultural product traceability service system design [J]. small and micro computer system, 2014, 35 (2): $261-265$.

[7] Liu Jia. Construction of agricultural product quality and safety traceability system based on two dimensional code [J]. Journal of Shandong Agricultural University (NATURAL SCIENCE EDITION), 2014, (5): 724-729.

[8] Bai Hongwu, Sun Aidong, Chen Jun, et al. Traceability system for quality and safety of agricultural products based on Internet of things [J]. Journal of agriculture and Agricultural Sciences, 2013, 29 (2): 415-420. 